OPEN ACCESS

Edited by:

Philip P. Foster,

Baylor College of Medicine,

United States

Reviewed by:

Federico Ranieri,

University of Verona, Italy

Ramona Ritzmann,

Clinic Rennbahn AG, Switzerland

*Correspondence:

James S. Thomas

jthomas32@vcu.edu

Received: 08 January 2020

Accepted: 13 July 2020

Published: 05 August 2020

Citation:

Stamenkovic $A$, van der Veen SM and Thomas JS (2020) Fear Priming:

A Method for Examining Postural

Strategies Associated With Fear

of Falling.

Front. Aging Neurosci. 12:241. doi: 10.3389/fnagi.2020.00241

\section{Fear Priming: A Method for Examining Postural Strategies Associated With Fear of Falling}

\author{
Alexander Stamenkovic ${ }^{1}$, Susanne M. van der Veen ${ }^{1,2}$ and James S. Thomas ${ }^{1,2 *}$ \\ ${ }^{1}$ Motor Control Laboratory, Department of Physical Therapy, Virginia Commonwealth University, Richmond, VA, \\ United States, ${ }^{2}$ Department of Physical Medicine and Rehabilitation, Virginia Commonwealth University, Richmond, VA, \\ United States
}

Fear of falling influences postural strategies used for balance, and is key in the maintenance of independent living and quality of life as adults age. However, there is a distinct need for methodology that aims to specifically address and prime fear under dynamic conditions, and to better determine the role of fear in movement preparation. This preliminary study investigated how fear priming influences fear of falling in young and older individuals, and assessed how changes in fear of falling map to movement behavior. Young $(21.5 \pm 1.7$ years, $n=10)$ and older $(58.1 \pm 2.2$ years $)$ participants matched for height, weight, and sex were repeatedly exposed to four different and incrementally challenging laboratory-based slipping perturbations during a self-initiated, goal-directed step and reach task. Both younger and older cohorts showed similar heightened perceptions in fear of falling after fear priming, and changes in peak joint excursions including reduced ankle flexion, and increased lumbar flexion after fear priming. Age-related changes were only evident in total mediolateral center of mass displacement, with younger participants showing greater displacement after fear priming. Despite clear differences in preparatory muscle onsets relative to reach onset seen in older participants, muscle timings or co-contraction indices were not significantly different. Methods utilizing repeated exposure to varying increases of a slipbased postural challenge can successfully prime fear of falling in individuals, regardless of age.

Keywords: fear of falling, posture, balance, fear priming, aging

\section{INTRODUCTION}

Fear of falling and movement anxiety related to postural threat have been shown to influence postural strategies used for balance, and are key factors in the maintenance of independent living and quality of life as adults age (Young and Williams, 2015). Generally, mechanisms underlying the role of fear in contributing to falls and stability are examined through two main experimental paradigms; using changes in height to induce fear (Adkin et al., 2002;

Abbreviations: $\mathrm{ABC}$, Activities of Balance Confidence scale; BoS, base of support; CCI, co-contraction index; CoM, center of mass; FES, Falls Efficacy scale; LB. "lubricant both" perturbation condition; LL, "lubricant landing" perturbation condition; LP, "lubricant plant" perturbation condition; NL, "no lubricant" perturbation condition; VAS, Visual Analog scale. 
Cleworth et al., 2012, 2016; Zaback et al., 2016), and by manipulating the expectation of an externally produced perturbation (Johnson et al., 2019). From these studies, a "stiffening strategy" characterized by increases in muscle coactivation (Nagai et al., 2012) and reduced ranges of motion and velocity of movement across joints of the lower limb (Brown et al., 2002) is associated with increased postural threat, with the goal of minimizing disturbances to the center of mass (CoM) position (Carpenter et al., 2001). Considering that the majority of falls occur during gaitbased events including weight bearing transitions and singlelimb support, it is less clear how fear of falling influences the interplay between postural-based movement and taskbased movement goals, as previous paradigms are predicated on feedback-based reactive mechanisms that aim to reduce postural changes. In fact, a number of activities, including gait and whole-body reaching, require preparatory postural adjustments across the lower limbs and trunk that drive displacement of the CoM for successful task performance (Brenière et al., 1987; Stapley et al., 1999; Leonard et al., 2009; Stamenkovic and Stapley, 2016).

When examined in the context of volitional movement control, fear of falling shows similar alterations in movement behaviors despite these differences in task goals. Additionally, fear of falling manifests in an impaired and "cautious" gait strategy (Delbaere et al., 2009) yet, it is unknown whether this is solely based on fear components or is multi-dimensional in nature as comparisons are often made between individuals exhibiting low or high fear of falling (Adkin et al., 2002; Nagai et al., 2012; Uemura et al., 2012). Furthermore, these paradigms adopt voluntary movements toward postures affording less stability (e.g., rise to toes - Adkin et al., 2002; Zaback et al., 2016, leg raises -Yiou et al., 2011; Gendre et al., 2016). These findings indicate that fear of falling can be characterized by a conscious prioritization of posture and minimization in CoM displacement. When we also consider age-related declines in postural control, either through the structural degradation of independent sensory processes (Manini et al., 2013) or multisensorimotor integration (Papegaaij et al., 2014), which may present as early as middle-age (Humes, 2015), methodology that incorporates increasing fear perceptions and examines movement preparation can provide insights into how fear influences the state of the sensorimotor system. As such, there is a distinct need for methodology that specifically addresses whether fear of falling perceptions can influence movement preparation and planning. Rather than focus on compensatory strategies to the application of an unexpected surface condition across high and low fear populations, the current paradigm aimed to investigate how priming fear (using known changes to surface condition) alters age-related preparatory movement strategies in an initially non-fearful population.

It was hypothesized that repeated exposure to varying degrees of a slipping perturbation would (1) prime state-specific fear in individuals and (2) alter age-related preparatory strategies that would prioritize balance maintenance, including minimizing CoM displacement through increased co-contraction and earlier activation of postural muscle activity relative to movement onset.

\section{METHODS}

\section{Participants}

Ten healthy young ( 5 female; mean age: $21.5 \pm 1.7$ years; mean height: $1.71 \pm 0.07 \mathrm{~m}$; mean weight $69.4 \pm 10.3 \mathrm{~kg}$ ) and 10 anthropometrically matched older participants ( 5 female; mean age: $58.1 \pm 2.2$ years; mean height: $1.69 \pm 0.09 \mathrm{~m}$; mean weight $69.6 \pm 9.12 \mathrm{~kg}$ ), without any known neurological, visual, or orthopedic impairments gave informed consent for all experimental procedures. Local institutional ethical approval (IRB \#13F014) was granted for all protocols and procedures. Assessment of initial perceptions surrounding balance confidence (Activities-Specific Balance Confidence scale - ABC), and fear of falling (via the Falls Efficacy scale - FES) found that participants in both cohorts reported having high balance confidence (ABC: YOUNG, $96.2 \%+5.1 \%$ vs. OLDER, $91.1 \%+6.4 \%)$ and low fear of falling (FES: YOUNG, $11.0 \%+1.6 \%$ vs. OLDER, $11.6 \%+3.5 \%)$. These were outside of mean scores generally associated with a greater predicted risk of falling (e.g., $<67 \%$ for ABC - Lajoie and Gallagher, 2004).

\section{Experimental Apparatus and Set-Up}

Participants stood barefoot on two linoleum covered tri-axial force plates (Model \#4060, Bertec, Columbus, OH, United States) that recorded ground reaction forces and moments at $1,000 \mathrm{~Hz}$ (Figure 1A). Whole-body kinematics were recorded using a custom designed marker set and 10-camera Vicon Nero system (Vicon, Oxford, United Kingdom) at $100 \mathrm{~Hz}$. Muscle activity for the trunk, lower limb and reaching arm were recorded using a 16-channel Trigno wireless surface electromyography (EMG) system (Delsys, Boston, MA, United States) at 2,000 Hz. EMG setup, including skin preparation followed procedures set forth in the Surface ElectroMyoGraphy for the Non-Invasive Assessment of Muscles guidelines (SENIAM; Hermens et al., 2000), while surface electrode (Trace1, Nikomed, Hatboro, PA, United States) placement aligned with SENIAM guidelines (Hermens et al., 2000), and previously identified sites for trunk musculature (Stamenkovic and Stapley, 2016). Kinematics, analog EMG, force place and target contact were synchronized through MotionMonitor (Innovative Sports, Chicago, IL, United States). Data collection occurred for a total of $8 \mathrm{~s}$.

\section{Experimental Procedures}

Participants were instructed to reach with their right hand "as fast as possible" while taking a step with their right foot, to complete a whole-body point-to-point movement. Movements were self-initiated with reach onset and offset determined using an infrared sensing system (FRK-030-M-Q8, Ramco Innovations, Des Moines, IA, United States) to identify initiation of hand movement, and contact with the target. Step and reach movements were made under 4 different surface-lubricating conditions created to elicit a greater likelihood of perturbation (e.g., through slipping and falling). The four conditions included no lubricant (NL), lubricant under the left planted leg (LP), under the right leg landing area (LL), and a combination of the prior two conditions, affecting both the postural and moving limbs 
A

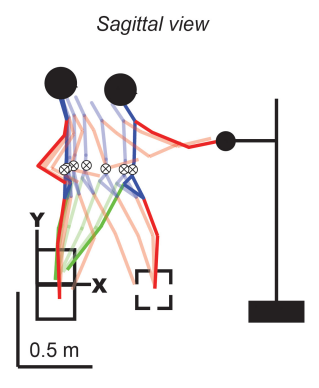

B

Calculated Variables

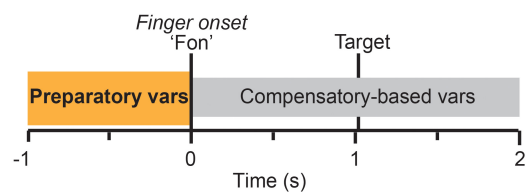

Step Characteristics

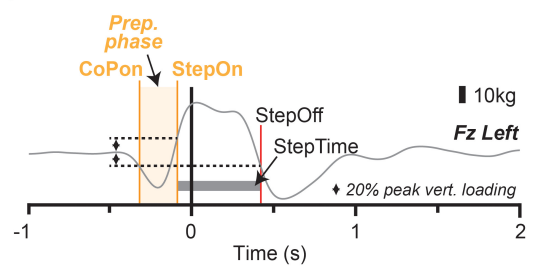

Base of Support (BoS) and Center of Mass (CoM)

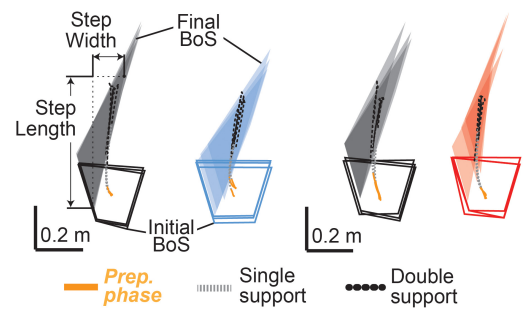

Muscle Activity

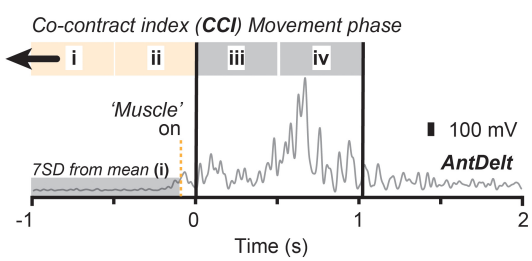

i Early $(-1.5 \mathrm{~s}:-0.5 \mathrm{~s})$

ii pre-Fon (-0.5s : 0s)

iii post-Fon (0s : $0.5 \mathrm{~s})$

iv pre-Target $(-0.5 \mathrm{~s}:$ Target)

Experimental Schema

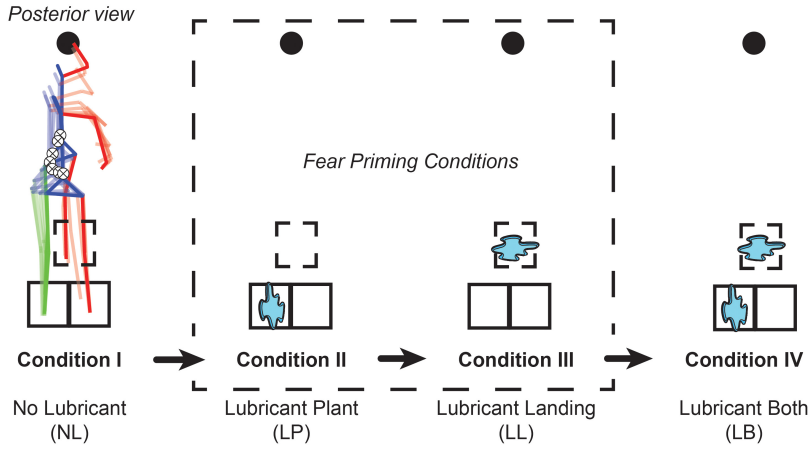

C

Representative participant muscle activity and joint excursions
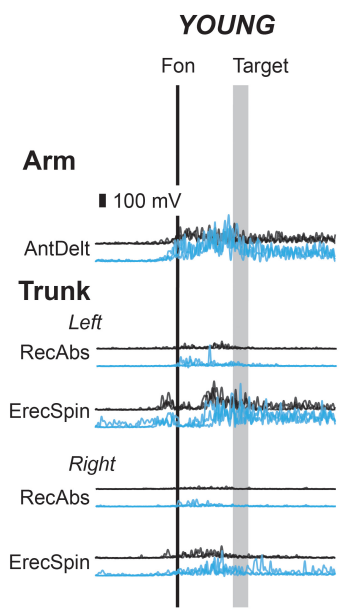

Landing Limb

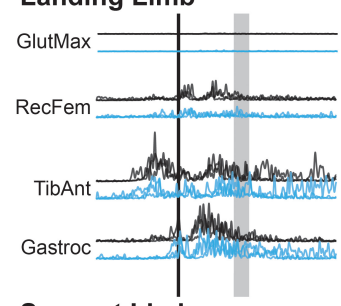

Support Limb

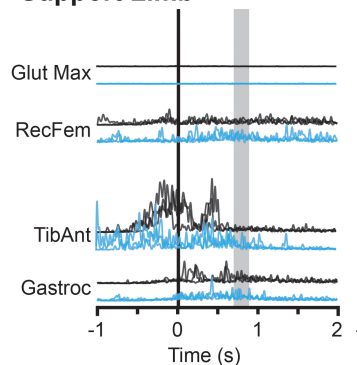

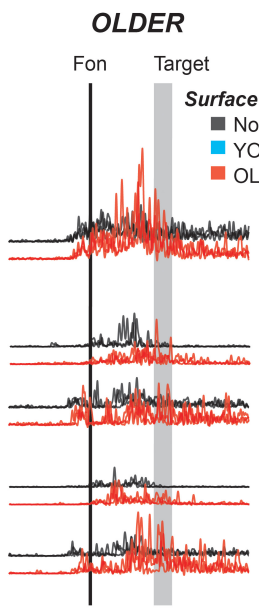
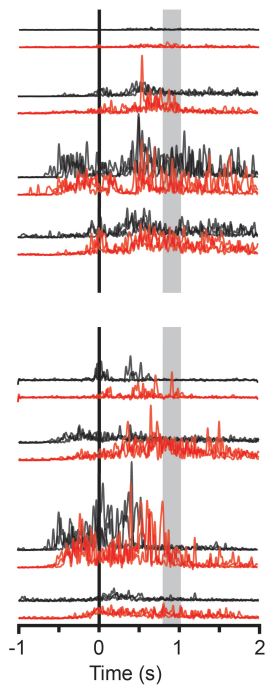

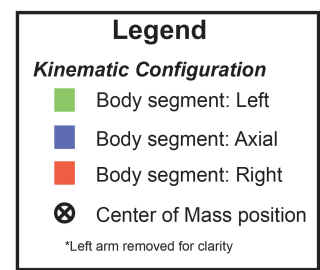

B)
Kinematic Configuration

Body segment: Left

Body segment: Right

( Center of Mass position

Leff arm removed for clarity

FIGURE 1 | Schematic of fear priming used in the current experimental protocol (A), with description of calculated movement preparation (orange) and compensatory-based variables (B), and representative examples of muscle activity, and joint excursions (C). (A) Participants began in a control condition without any slipping perturbation (Condition I - NL) and progressed through to Condition IV (LB) where slipping perturbation was present under both the supporting limb ("plant") and within the step landing position. (B) Calculated variables of interest derived from ground reaction forces (i.e., step characteristics), kinematics (center of mass, $\mathrm{CoM}$ ), and muscle activity (i.e., muscle onsets and co-contraction indexes, CCl). Variables associated with movement preparation are termed preparatory and highlighted in orange. (C) Representative muscle activity and joint excursions prior to movement onset (i.e., Fon), and during the step-and-reach task. Control trials (i.e., Condition I, NL) are depicted in black, with perturbation trials (i.e., Condition IV - LB) for YOUNG and OLDER participants in blue and red, respectively. 
(LB). Target position was based on standardized measures of hip height (from ground), trunk length, and arm length to produce a vertical target position that required $15^{\circ}$ of trunk flexion (Thomas et al., 2008) with the anterior-posterior distance of the target increased by an additional $50 \%$ of total hip height to ensure a step was required (Figure 1A). Trials $(n=3)$ for each perturbation condition were presented in a blocked fashion (NL, LP, LL, LB) with the intent to provide progressively greater challenge under which reaching movements were made and heighten overall perceptions of fall risk. Participants were familiarized with the task and speed necessary in a short practice session prior to data collection.

\section{Data Analysis}

Analysis was completed offline using Matlab (ver. R2018, The Mathworks, Natick, MA, United States). Kinematic data were smoothed using a 40-point Savitzky-Golay filter and DC offset removed. Analog EMG signals were amplified $(1,000 \times)$, notch filtered $(60 \mathrm{~Hz})$ before being de-meaned, rectified and low-pass filtered using a 4th order zero-lag Butterworth at $100 \mathrm{~Hz}$. EMG was normalized to the maximum amplitude recorded across all conditions per individual and muscle.

Prior to each condition, two $100 \mathrm{~mm}$ Visual Analog scales (VASs) were used to assess state-specific fear of falling, regarding expectations of participant's "likelihood of falling" and "concern of falling." Figure 1B highlights step and CoM based characteristics, and temporal and amplitude-based muscle activity related to movement preparation (Figure 1B: orange). Preparatory anteroposterior (AP) and mediolateral (ML) CoM acceleration and excursion measures were determined using the onset of center of pressure (Figure 1B: CoPon), and landing limb unloading (i.e., step onset, Figure 1B: StepOn). Further, timing of trunk and lower limb muscle activity were calculated relative to the initiation of reach. Muscle onsets were determined using a custom algorithm based on the maintenance of muscle activity for $50 \mathrm{~ms}$ beyond $7 \mathrm{SD}$ of average baseline activity and confirmed via visual inspection (Teasdale et al., 1993). Measures of muscle amplitude were determined using the ratio of agonist to antagonist activation, or co-contraction index (CCI; Craig et al., 2016). The CCI was divided into four distinct phases; (i) early activity 1.5 to $0.5 \mathrm{~s}$ prior to reach onset, (ii) preparatory activity $0.5 \mathrm{~s}$ prior to reach onset, (iii) movement activity $0.5 \mathrm{~s}$ following reach onset, and (iv) termination activity $0.5 \mathrm{~s}$ prior to target contact.

To characterize how changes in fear of falling mapped to movement behavior changes in step characteristics including AP distance between malleoli of the support and landing limb (StepLength), ML distance between malleoli of the support and landing limb (StepWidth), and time between step onset and offset (StepTime), as well as peak-to-peak joint and CoM excursions across the duration of movement were also analyzed (Figure 1C).

\section{Statistical Analysis}

The effect of fear on movement behaviors were assessed using separate two-way mixed repeated measures ANOVAs (Condition $\times$ AgeGroup) using SPSS (version 25, IBM, Armonk, NY, United States). To address our primary outcomes, repeated measures ANOVAs on changes in state-specific fear (Dependent variables, DV: VAS "Likely to fall," VAS "Concerned about falling"), were followed by repeated measures ANOVAs for preparatory variables including step characteristics (DV: CoPon, StepOn), CoM characteristics (DV: preparatory CoM AP/ML accelerations and excursions), muscle onsets (DV: see muscle list), and co-contraction indices (DV: CCI i, CCI ii). Secondary analyses investigated additional movement behavior metrics including compensatory changes in step characteristics (DV: StepOff, StepTime, StepLength, StepWidth), CoM excursions (DV: CoM total AP excursion, CoM total ML excursion), muscle co-contraction indices (DV: CCI iii, CCI iv), and peak joint excursions (DV: ankle, knee, hip, lumbar, thorax, shoulder, elbow) associated with fear of falling. BonferroniHolm adjustments were applied to main repeated measures ANOVA results to reduce the family-wise error rate before determining significance across related measures (i.e., step or CoM characteristics, muscle onsets, CCI, joint excursions). This was achieved by altering the initial level of significance $(p<0.05)$ with respect to the total number of tests performed to produce a more conservative significance level such that; $p=0.05 /(\#$ DVs $\times 2$ main effect/interactions). GreenhouseGeisser adjustments were made in cases where violations of sphericity were observed. When applicable, further post hoc analyses were conducted with Bonferroni adjustment for multiple pairwise comparisons.

\section{RESULTS}

Descriptive statistics and results from individual repeated measures ANOVAs are available in tabular form (see Supplementary Material).

\section{Influence of Fear Priming on State-Specific Fear of Falling}

Figure 2A shows the changes in fear perceptions regarding falling as participants made step and reach movements across increasingly challenging surface conditions. A main effect of surface condition was seen for both VAS regarding state-specific expectations about falling ["Likely": $F_{(1.845,29.518)}=36.463$, $p<0.001, \eta^{2} p=0.695$; “Concerned": $F_{(3,48)}=31.343, p<0.001$, $\left.\eta_{p}^{2}=0.662\right]$. Post hoc analyses revealed that both YOUNG and OLDER cohorts had heightened perceptions of falling in the LB surface condition relative to the control (NL) condition ("Likely" - YOUNG: $p=0.015$; OLDER: $p=0.001$; "Concerned" YOUNG: $p=0.007$; OLDER: $p=0.004$ ).

\section{Characterizing Movement Behavior: Movement Preparation Stepping and CoM Characteristics}

Figure 2B shows changes in stepping and CoM characteristics prior to, and following movement onset. Variables highlighted in orange represent those that were preparatory in nature (for visual representation see Figure 1B). Following Bonferroni-Holm adjustments, only CoM acceleration during the preparatory 

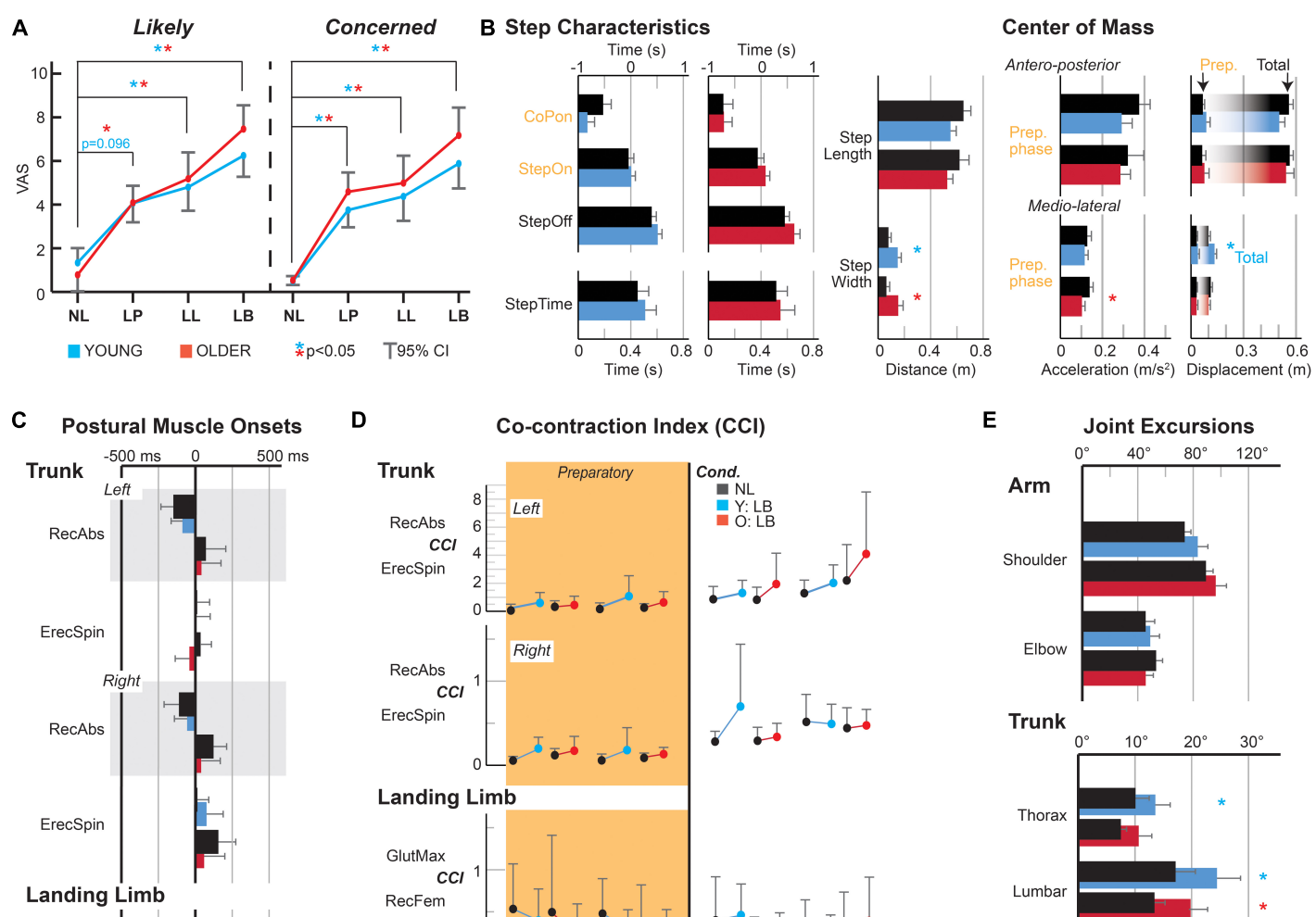

D

Co-contraction Index (CCl)

E

Trunk
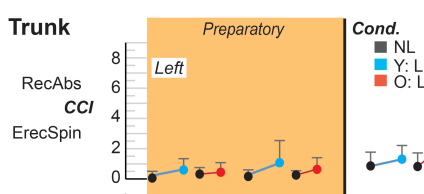

$=\mathrm{NL}$
$\mathrm{Y}: \mathrm{LB}$
$\mathrm{O}: \mathrm{LB}$

ErecSpin

${ }_{0}^{2}[0.5 \cdot 0$
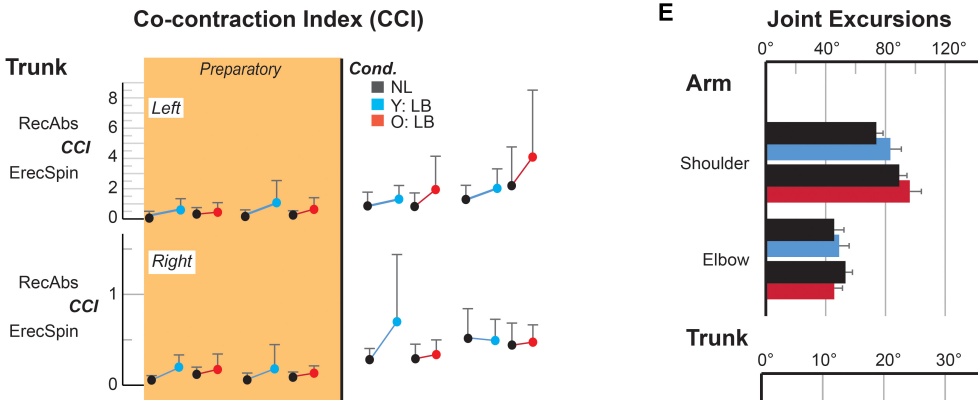

Landing Limb

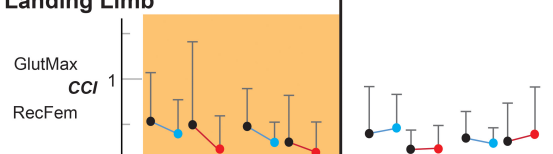

Trunk

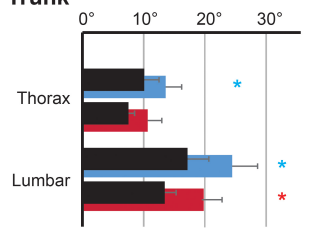

Landing Limb
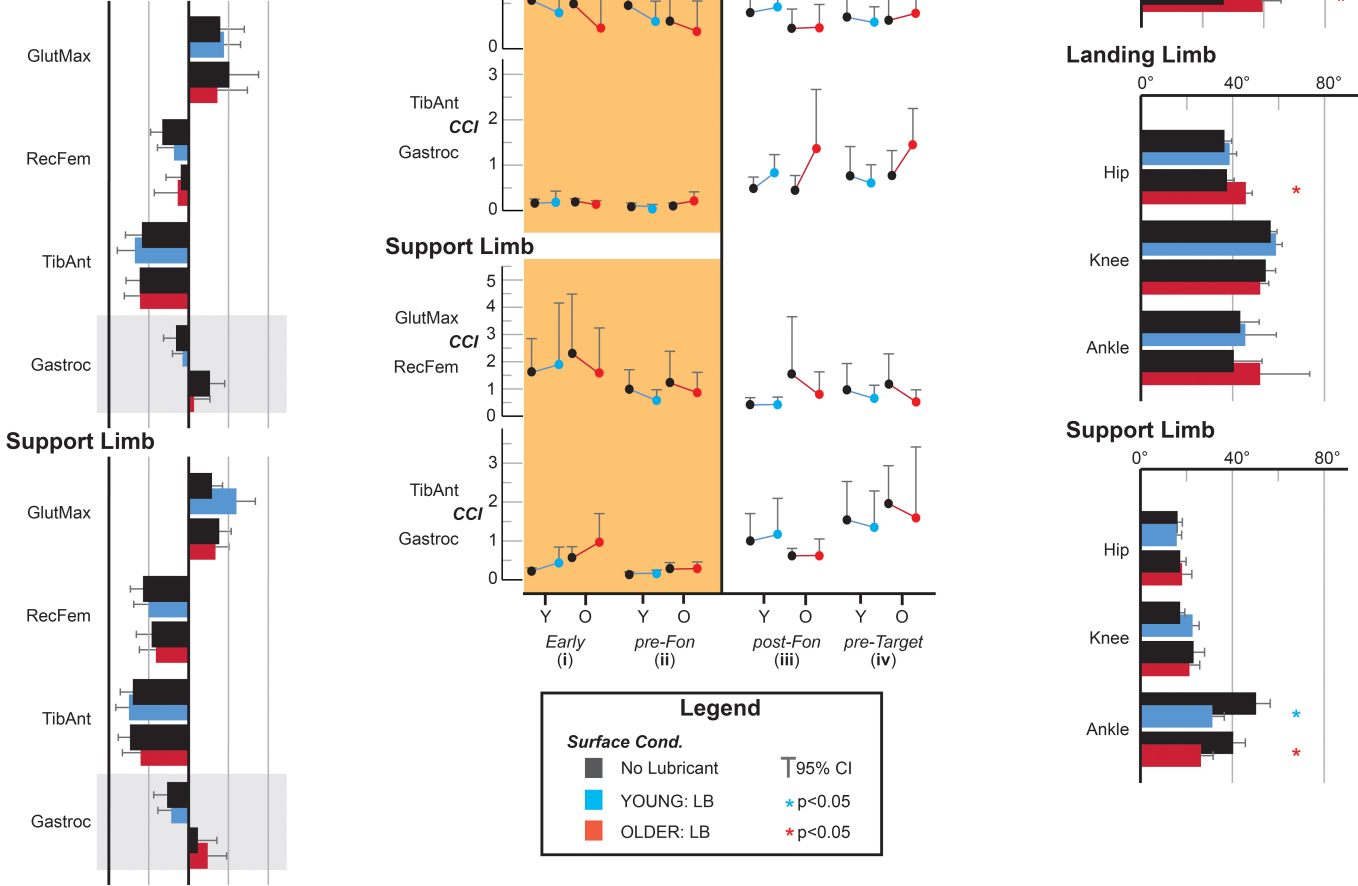

Support Limb
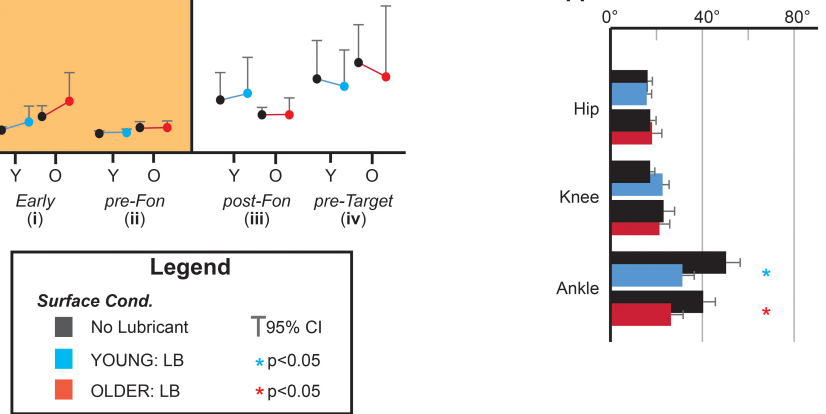

FIGURE 2 | Comparison of fear cognitions (A), step characteristics and center of mass measures (B), muscle onsets (C), muscle co-contraction indexes (CCI) (D), and joint excursions (E) across fear conditions in YOUNG (blue) and OLDER (red) participants. (A) Visual Analog scale (VAS) outcomes on self-reported perceptions on the likelihood of falling ("Likely") and concern about falling ("Concerned") revealed an incremental increase in fear cognitions across fear priming conditions regardless of age group. (B) Step-based and center of mass (CoM) movement characteristics revealed differential changes on preparatory and compensatory movement behaviors. (C) Muscle onsets for postural muscles of the trunk and lower limbs. While onsets did not significantly vary across surface condition or age, a number of muscles (shown in gray) showed delayed activations following movement onset in OLDER participants. (D) Muscle CCl across preparatory (i, ii - orange) and compensatory movement phases (iii, iv). No significant differences in CCl measures were seen across surface condition or age. (E) Peak-to-peak joint excursions for the reaching arm, trunk, and lower limbs. Lumbar excursions were increased and, ankle excursions in the supporting limb were decreased in both cohorts as a result of surface condition. All values reported as mean $+95 \%$ confidence interval. ${ }^{*} p<0.005$ (Bonferroni-Holm adjusted). 
phase showed a significant main effect of surface condition $\left[F_{(1,18)}=11.06, p=0.004, \eta^{2} p=0.381\right]$.

\section{Muscle Activity}

Clear preparatory activity was seen in the trunk and lower limbs, particularly within the erector spinae and tibialis anterior (see Figure 1C for representative example). When pooled, analyses revealed no differences in muscle onsets (Figure 2C) or preparatory amplitude measures (i.e., CCI) in the "early" and "preparatory" movement phases (see Figure 2D, orange background). However, despite a lack of differences in muscle onsets, a clear distinction in the onset of certain muscles was seen between age groups. Specifically, YOUNG participants displayed bilateral activity of rectus abdominis and gastrocnemius that preceded movement onset, while these muscles often activated following the initiation of reach in OLDER participants (Figure 2C, gray boxes). Surprisingly, these timings did not alter as a consequence of condition.

\section{Characterizing Movement Behavior: Compensatory Strategies \\ Stepping and CoM Characteristics}

A number of step and CoM measures showed differences following movement onset (Figure 2B). Of note, an interaction effect was present for total ML CoM excursion $\left[F_{(1,18)}=11.630\right.$, $\left.p=0.003, \eta^{2} p=0.392\right]$. Post hoc analysis revealed that this was driven by greater excursion in YOUNG participants $(p=0.001)$. By extension, step width showed a main effect of surface condition $\left[F_{(1,18)}=19.020, p<0.001, \eta_{p}^{2}=0.514\right]$, increasing in both YOUNG and OLDER participants.

\section{Muscle Activity}

Analysis of CCI during compensatory phases of movement (i.e., Figure 2D, CCI iii, CCI iv) did not show any differences across surface condition or age group.

\section{Joint Excursions}

Changes in surface condition influenced peak to peak joint kinematics (see Figure 1C for representative example and Figure 2E for pooled means), resulting in similar increases in lumbar flexion $\left[F_{(1,18)}=23.74, p<0.001, \eta_{p}^{2}=0.597\right]$, and reductions in support limb ankle flexion $\left[F_{(1,18)}=22.56\right.$, $\left.p<0.001, \eta_{p}^{2}=0.585\right]$ across age groups respectively. Greater thoracic flexion $\left[F_{(1,18)}=10.77, p=0.005, \eta_{p}^{2}=0.402\right]$ and leading limb hip flexion $\left[F_{(1,18)}=10.29, p=0.005, \eta^{2} p=0.391\right]$ was seen as a consequence of surface condition.

\section{DISCUSSION}

The present study examined the ability of a perturbation-based step and reach paradigm to elicit fear and characterize how such fear cognitions shape movement preparation behavior. By gradually exposing participants to changes in surface condition, the paradigm was successfully able to produce increases in perceived fear of falling (Figure 2A). The increases in statespecific fear in the current study lie in contrast to previous findings, where unpredictable postural perturbations initially increase anxiety, which significantly reduces following repeated exposure (Johnson et al., 2019). However, it is unclear whether this is related to the type of perturbation produced. Exposure to slipping-specific perturbations have been associated with recovery strategies that adapt at similar rates to result in fewer falls between younger and older adults, although fear was not recorded in these studies (Pavol et al., 2002; Pai et al., 2007). By producing perceptions of fear of falling in a cohort of initially non-fearful younger and older adults, the current paradigm also induced changes in compensatory stepping strategies and peak joint excursions. Specifically, OLDER participants showed a reduction in total ML CoM excursion with shorter and wider steps that followed (rather than preceded) movement of the reaching arm during the more fearful LB condition. In fact, evidence of an attempt to minimize ML CoM excursion (rather than increase, as seen in the YOUNG cohort) supports the adoption of a balance-centric postural strategy, as lateral instability is associated with an increased fall risk in older adults (Rogers and Mille, 2003). Similar to expectations from previous feedback-based paradigms assessing fear of falling, both YOUNG and OLDER participants displayed reductions in ankle excursion indicative of the adoption of a stiffening strategy. Considering the lack of alterations in arm kinematics, the greater excursions of the trunk that complemented changes in ankle excursion are most likely a consequence of motor equivalence in order to successfully achieve task goals (i.e., target contact). Whether this attribute of the current protocol can be leveraged in the future assessment of the link between fear cognitions and balance performance is unclear, however both step characteristics and trunk kinematics are shown to be important predictors of falls and recovery step after lab-induced trips, and highly implicated in falls risk (Marone et al., 2011).

Despite changes in fear influencing step characteristics and joint excursions across the movement, similar alterations were not reflected in measures of underlying muscle activity. While age-related differences in muscle onsets showed delays in the production of older adults preparatory postural adjustments, these were not affected by fear priming. Further, the impact of fear priming was not observable in the timing or muscle co-contraction index (i.e., CCI) across the trunk and lower limbs. Considering that increases in co-contraction are often found when examining fear-related changes that occur with postural control (Nagai et al., 2012) it is surprising that these were not replicated in the current study, especially during preparatory periods preceding movement onset. As all conditions required a step to achieve the task, it is plausible that increases in co-contraction, especially around the ankle joint would have impeded task completion by making it more difficult to initiate a step. Therefore, a generalized movement preparation strategy was adopted, despite greater concerns regarding the likelihood of falling. Additionally, the discrepancy between falls efficacy (via FES) and state-specific fear of falling (via VAS) may provide a conceptual explanation to the broad lack of changes in preparatory strategies seen in the current study. Hadjistavropoulos et al. (2011) argue that both are separate constructs that contribute to fall outcomes and impact 
on balance performance. Therefore, a combination of high efficacy in one's ability to maintain balance, and task requirements (i.e., promoting movement or maintaining balance) may mediate changes to preparatory postural alterations that would often follow increases in high contextual anxiety and fear of falling, particularly if concerns regarding falling align with the situational likelihood of a fall occurring (i.e., that an individual accurately appraises the "current" situation). In fact, the mechanism underlying this interaction is unclear, with work investigating neural correlates responsible for the specific interaction of fear of falling on movement behavior limited. As stiffening strategies adopted in static perturbations and cautious gait are thought to represent a shift to a conscious and cortical-based control of posture, frontoparietal interactions provide a viable conduit for investigation. In fact, decision confidence (Bang and Fleming, 2018), fear conditioning and extinction (Giustino and Maren, 2015), and executive behavior planning (particularly with respect to the future consequences of actions - Matsumoto and Tanaka, 2004) have all been implicated with medial prefrontal cortex function. Only a single recent investigation has provided evidence that individuals with generalized high fear of falling have greater neural inefficiency (i.e., higher brain activations associated with decrements in performance) in the pre-frontal cortex, however this occurs when a concomitant cognitive process (i.e., dual-task) is applied (Holtzer et al., 2019). Whether this applies purely to fear perceptions without confounding factors is unknown, however future examinations could leverage the current paradigm to parse out such influences on movement preparation, especially considering that planning actions between predictable and unpredictable events are thought to have distinct neural substrates within the pre-frontal cortex (Koechlin et al., 2000).

\section{Study Limitations}

While the possible effects of condition order cannot be discounted, perturbation conditions were deliberately presented in a blocked fashion with the intent to provide progressively greater challenge under which reaching movements were made, thus heightening overall perceptions of fall risk. This was based on a similar framework upon which graded exposure paradigms operate (Trost et al., 2009). While this makes it challenging to parse motor learning effects from those of fear of falling, we would consider that fear conditioning would be modulated (i.e., extinguished) by improvements based on motor learning and knowledge of continued task success, rather that the maintenance (and general increase of fear perceptions) seen in the current study.

Further, as general safety and feasibility of the paradigm was a key consideration in this preliminary study we deliberately recruited OLDER adults between 55 and 65 which is relatively young compared to the literature (i.e., 65+ years old). However, our differences in CoM excursion and preparatory sequences of muscle activity highlight that changes in movement behavior are detectable even within a middle-aged cohort. Considering the focus on older cohorts (65+ years old) within the literature, this highlights the importance of emphasizing the investigation of a greater spectrum of age across human development, especially in the context of understanding mechanisms surrounding fear of falling influences on postural control (Hadjistavropoulos et al., 2011).

\section{CONCLUSION}

The current paradigm shows that fear priming, and repeated exposure to progressively increasing perturbations that challenge balance, have the capacity to increase fear of falling in an initially non-fearful cohort of younger and older adults. As such, this study provides a novel assessment of how fear conditioning changes motor behavior in a healthy cohort and provides a method to examine how fear changes motor planning in individuals.

\section{DATA AVAILABILITY STATEMENT}

The datasets generated for this study are available on request to the corresponding author.

\section{ETHICS STATEMENT}

The studies involving human participants were reviewed and approved by the Ohio University Institutional Review Board (IRB \#13F014). The patients/participants provided their written informed consent to participate in this study.

\section{AUTHOR CONTRIBUTIONS}

JT conceived and designed the study, and collected the data. AS and SV performed the analysis. AS produced the first draft of the manuscript. All authors contributed to the interpretation of data, critical review of the manuscript, and approval of the final version of this manuscript.

\section{FUNDING}

AS was supported by NIH R01AT006978. JT was supported by NIH R01AT006978 and R01HD088417. AS and SV were supported by a NIH R01HD088417.

\section{SUPPLEMENTARY MATERIAL}

The Supplementary Material for this article can be found online at: https://www.frontiersin.org/articles/10.3389/fnagi. 2020.00241/full\#supplementary-material 


\section{REFERENCES}

Adkin, A., Frank, J., Carpenter, M., and Peysar, G. (2002). Fear of falling modifies anticipatory postural control. Exp. Brain Res. 143, 160-170. doi: 10.1007/ s00221-001-0974-8

Bang, D., and Fleming, S. M. (2018). Distinct encoding of decision confidence in human medial prefrontal cortex. PNAS 115, 6082-6087. doi: 10.1073/pnas. 1800795115

Brenière, Y., Do, M. C., and Bouisset, S. (1987). Are dynamic phenomena prior to stepping essential to walking? J. Motor Behav. 19, 62-76. doi: 10.1080/ 00222895.1987.10735400

Brown, L., Gage, W., Polych, M., Sleik, R., and Winder, T. (2002). Central set influences on gait. Exp. Brain Res. 145, 286-296. doi: 10.1007/s00221-0021082-0

Carpenter, M., Frank, J., Silcher, C., and Peysar, G. (2001). The influence of postural threat on the control of upright stance. Exp. Brain Res. 138, 210-218. doi: $10.1007 / \mathrm{s} 002210100681$

Cleworth, T. W., Chua, R., Inglis, J. T., and Carpenter, M. G. (2016). Influence of virtual height exposure on postural reactions to support surface translations. Gait Posture 47, 96-102. doi: 10.1016/j.gaitpost.2016.04.006

Cleworth, T. W., Horslen, B. C., and Carpenter, M. G. (2012). Influence of real and virtual heights on standing balance. Gait Posture 36, 172-176. doi: 10.1016/j. gaitpost.2012.02.010

Craig, C. E., Goble, D. J., and Doumas, M. (2016). Proprioceptive acuity predicts muscle co-contraction of the tibialis anterior and gastrocnemius medialis in older adults' dynamic postural control. Neuroscience 322, 251-261. doi: 10. 1016/j.neuroscience.2016.02.036

Delbaere, K., Sturnieks, D. L., Crombez, G., and Lord, S. R. (2009). Concern about falls elicits changes in gait parameters in conditions of postural threat in older people. J. Gerontol. Ser. A 64A, 237-242. doi: 10.1093/gerona/gln014

Gendre, M., Yiou, E., Gélat, T., Honeine, J.-L., and Deroche, T. (2016). Directional specificity of postural threat on anticipatory postural adjustments during lateral leg raising. Exp. Brain Res. 234, 659-671. doi: 10.1007/s00221-015-4471-x

Giustino, T. F., and Maren, S. (2015). The role of the medial prefrontal cortex in the conditioning and extinction of fear. Front. Behav. Neurosci. 9:298. doi: 10.3389/fnbeh. 2015.00298

Hadjistavropoulos, T., Delbaere, K., and Fitzgerald, T. D. (2011). Reconceptualizing the role of fear of falling and balance confidence in fall risk. J. Aging Health 23, 3-23. doi: 10.1177/0898264310378039

Hermens, H. J., Freriks, B., Disselhorst-Klug, C., and Rau, G. (2000). Development of recommendations for sEMG sensors and sensor placement procedures. J. Electromyogr. Kinesiol. 10, 361-374. doi: 10.1016/s1050-6411(00) 00027-4

Holtzer, R., Kraut, R., Izzetoglu, M., and Ye, K. (2019). The effect of fear of falling on prefrontal cortex activation and efficiency during walking in older adults. GeroScience 41, 89-100. doi: 10.1007/s11357-019-00056-4

Humes, L. E. (2015). Age-related changes in cognitive and sensory processing: focus on middle-aged adults. Am. J. Audiol. 24, 94-97. doi: 10.1044/2015_aja14-0063

Johnson, K. J., Zaback, M., Tokuno, C. D., Carpenter, M. G., and Adkin, A. L. (2019). Repeated exposure to the threat of perturbation induces emotional, cognitive, and postural adaptations in young and older adults. Exp. Gerontol. 122, 109-115. doi: 10.1016/j.exger.2019.04.015

Koechlin, E., Corrado, G., Pietrini, P., and Grafman, J. (2000). Dissociating the role of the medial and lateral anterior prefrontal cortex in human planning. PNAS 97, 7651-7656. doi: 10.1073/pnas.130177397

Lajoie, Y., and Gallagher, S. P. (2004). Predicting falls within the elderly community: comparison of postural sway, reaction time, the Berg balance scale and the Activities-specific Balance Confidence (ABC) scale for comparing fallers and non-fallers. Arch. Gerontol. Geriatr. 38, 11-26. doi: 10.1016/s01674943(03)00082-7

Leonard, J. A., Brown, R. H., and Stapley, P. J. (2009). Reaching to multiple targets when standing: the spatial organization of feedforward postural adjustments. J. Neurophysiol. 101:2120. doi: 10.1152/jn.91135.2008
Manini, T. M., Hong, S. L., and Clark, B. C. (2013). Aging and muscle: a neuron's perspective. Curr. Opin. Clin. Nutr. Metab. Care 16, 21-26.

Marone, J. R., Rosenblatt, N. J., Troy, K. L., and Grabiner, M. D. (2011). Fear of falling does not alter the kinematics of recovery from an induced trip: a preliminary study. Arch. Phys. Med. Rehabil. 92, 2093-2095. doi: 10.1016/j. apmr.2011.06.034

Matsumoto, K., and Tanaka, K. (2004). The role of the medial prefrontal cortex in achieving goals. Curr. Opin. Neurobiol. 14, 178-185. doi: 10.1016/j.conb.2004. 03.005

Nagai, K., Yamada, M., Uemura, K., Tanaka, B., Mori, S., Yamada, Y., et al. (2012). Effects of fear of falling on muscular coactivation during walking. Aging Clin. Exp. Res. 24, 157-161.

Pai, Y., Espy, D., Bhatt, T., Wang, E., and Pavol, M. (2007). Age does not reduce one's ability to adapt and resist backward falling following repeated slip exposure. J. Geriatr. Phys. Ther. 30, 138-139. doi: 10.1519/00139143200712000-00018

Papegaaij, S., Taube, W., Baudry, S., Otten, E., and Hortobágyi, T. (2014). Aging causes a reorganization of cortical and spinal control of posture. Front. Aging Neurosci. 26:28. doi: 10.3389/fnagi.2014.00028

Pavol, M. J., Runtz, E. F., Edwards, B. J., and Pai, Y.-C. (2002). Age influences the outcome of a slipping perturbation during initial but not repeated exposures. J. Gerontol. Ser. A Biol. Sci. Med. Sci. 57:M496.

Rogers, M., and Mille, M. (2003). Lateral stability and falls in older people. Exerc. Sport Sci. Rev. 31, 182-187. doi: 10.1097/00003677-200310000-00005

Stamenkovic, A., and Stapley, P. (2016). Trunk muscles contribute as functional groups to directionality of reaching during stance. Exp. Brain Res. 234, 11191132. doi: 10.1007/s00221-015-4536-x

Stapley, P. J., Pozzo, T., Cheron, G., and Grishin, A. (1999). Does the coordination between posture and movement during human whole-body reaching ensure center of mass stabilization? Exp. Brain Res. 129, 134-146. doi: 10.1007/ s002210050944

Teasdale, N., Bard, C., Fleury, M., Young, D. E., and Proteau, L. (1993). Determining movement onsets from temporal series. J. Motor Behav. 25, 97-106. doi: 10.1080/00222895.1993.9941644

Thomas, S. J., France, R. C., Lavender, A. S., and Johnson, R. M. (2008). Effects of fear of movement on spine velocity and acceleration after recovery from low back pain. Spine 33, 564-570. doi: 10.1097/brs.0b013e3181657f1a

Trost, Z., France, C. R., and Thomas, J. S. (2009). Examination of the photograph series of daily activities (PHODA) scale in chronic low back pain patients with high and low kinesiophobia. Pain 141, 276-282. doi: 10.1016/j.pain.2008.11.016 Uemura, K., Yamada, M., Nagai, K., Tanaka, B., Mori, S., and Ichihashi, N. (2012). Fear of falling is associated with prolonged anticipatory postural adjustment during gait initiation under dual-task conditions in older adults. Gait Posture 35, 282-286. doi: 10.1016/j.gaitpost.2011.09.100

Yiou, E., Deroche, T., Do, M., and Woodman, T. (2011). Influence of fear of falling on anticipatory postural control of medio-lateral stability during rapid leg flexion. Eur. J. Appl. Physiol. 111, 611-620. doi: 10.1007/s00421-010-1680-7

Young, W. R., and Williams, A. M. (2015). How fear of falling can increase fall-risk in older adults: applying psychological theory to practical observations. Gait Posture 41, 7-12. doi: 10.1016/j.gaitpost.2014.09.006

Zaback, M., Carpenter, M. G., and Adkin, A. L. (2016). Threat-induced changes in attention during tests of static and anticipatory postural control. Gait Posture 45, 19-24. doi: 10.1016/j.gaitpost.2015.12.033

Conflict of Interest: The authors declare that the research was conducted in the absence of any commercial or financial relationships that could be construed as a potential conflict of interest.

Copyright (c) 2020 Stamenkovic, van der Veen and Thomas. This is an open-access article distributed under the terms of the Creative Commons Attribution License (CC BY). The use, distribution or reproduction in other forums is permitted, provided the original author(s) and the copyright owner(s) are credited and that the original publication in this journal is cited, in accordance with accepted academic practice. No use, distribution or reproduction is permitted which does not comply with these terms. 